\title{
El papel de las percepciones sociales en el impacto de programas de conservación
}

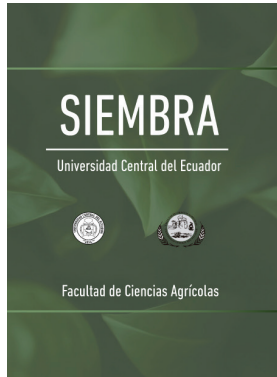

\section{The role of social perceptions in the impact of conservation programs}

\author{
Susana Ortega López ${ }^{1}$, Denise Soares de Moraes $^{2}$
}

\footnotetext{
${ }^{1}$ Instituto Mexicano de Tecnología del Agua (IMTA), Coordinación de Hidrología. Blvd. Paseo Cuauhnáhuac 8532, Progreso, 62550 Jiutepec, Morelos, México.

$\bowtie$ ortegasusy2@hotmail.com

(D. https://orcid.org/0000-0002-0393-2087

${ }^{2}$ Instituto Mexicano de Tecnología del Agua (IMTA), Coordinación de comunicación, Participación e Información. Blvd. Paseo Cuauhnáhuac 8532, Progreso, 62550 Jiutepec, Morelos, México.

$\bowtie$ denisefsoares@yahoo.com.mx

(Q) https://orcid.org/0000-0003-1811-0139

*Autor de correspondencia:

ortegasusy2@hotmail.com
}

\section{Resumen}

Con esta contribución se propone identificar y analizar cómo los actores sociales locales se relacionan con sus bosques y perciben los efectos de acciones de conservación de suelos y agua llevadas a cabo en la región, con el objetivo de generar recomendaciones para el diseño y puesta en práctica de acciones más asertivas en cuanto a las necesidades y demandas locales. El estudio se llevó a cabo en la microcuenca Ichupio, perteneciente a la cuenca del lago de Pátzcuaro, en el estado de Michoacán, México. Los resultados indican potenciales conflictos entre comunidades por el uso de los bosques, la ausencia de organización social para el establecimiento de un plan de manejo forestal sostenible, así como observaciones por parte de la población local a la implementación de acciones de conservación. Se concluye argumentando acerca de la necesidad de replantear las estrategias sobre las acciones de conservación, desde una territorialización efectiva, a fin de atacar las causas de la problemática y no solamente sus síntomas, para que puedan realmente cumplir con su mandato de recuperar y conservar la base de recursos naturales.

Palabras clave: bosques, percepciones, conservación, organización social.

\section{SIEMBRA}

https://revistadigital.uce.edu.ec/index.php/SIEMBRA e-ISSN: $2477-8850$

\section{ISSN: $1390-8928$}

Periodicidad: semestral

vol. 9, núm. 1, 2022

siembra.fag@uce.edu.ec

DOI: https://doi.org/10.29166/siembra.v9i1.3072

Esta obra está bajo una licencia internacional Creative Commons Atribución-NoComercial

\begin{abstract}
This contribution proposes to identify and analyze how local social actors relate to their forests and perceive the effects of soil and water conservation actions carried out in the region, in order to generate recommendations for the design and implementation of more assertive actions regarding local needs and demands. The study was carried out in the Ichupio micro-basin, belonging to the Lake Pátzcuaro basin, Michoacán state, México. The results indicate potential conflicts between communities over forests use, the absence of social organization for the establishment of a sustainable forest management plan, as well as observations by the local population, to the implementation of conservation actions. It concludes by arguing about the need to rethink the strategies of conservation actions, from an effective territorialization, in order to attack the causes of the problem and not only its symptoms, so that they can really fulfill their mandate to recover and preserve the base of natural resources.
\end{abstract}

Keywords: forests, perceptions, conservation, social organization. 


\section{Introducción}

Los bosques proporcionan múltiples beneficios y servicios ambientales, sociales y económicos, que contribuyen al desarrollo de la sociedad; proveen servicios hidrológicos, captura de carbono, madera, productos forestales no maderables (PFNM), son hábitat de flora y fauna, además de ser esenciales para el mantenimiento de la biodiversidad y adaptación al cambio climático. Los bosques circundantes a las grandes zonas urbanas proveen una serie de servicios ambientales, sin los cuales difícilmente éstas existirían; sin embargo, la presión de los asentamientos humanos, conforme la población se incrementa, incide directamente sobre la estabilidad ecológica de los ecosistemas. Por lo cual, mantener la cubierta forestal en México constituye un verdadero desafío, considerando las necesidades presentes y futuras de una población de 126 millones de habitantes (Comisión Nacional Forestal [CONAFOR], 2019; Dodds et al., 2013; Instituto Nacional de Estadística y Geografía [INEGI], 2020).

En México existe una serie de problemáticas en torno a los bosques, que inciden directamente en su deterioro y poseen un origen multifacético relacionado, entre otros, con disputas por el poder territorial, crecimiento industrial y poblacional, carencia de planeación ambiental del desarrollo, corrupción y debilidad institucional. Según Monforte y Cantú (2009) el papel de los bosques en la regulación del ciclo hidrológico es fundamental, dado que éste se equilibra por medio de dos zonas: recarga y descarga, las cuales deben estar en armonía, es decir, la descarga no puede ser mayor que la recarga. El desequilibrio del ciclo hidrológico tiene su origen en el aprovechamiento social de la naturaleza, por generar procesos de deforestación que reducen la infiltración en los mantos freáticos y una extracción de mayor cantidad de agua que la que se recarga. Al respecto, en el periodo 2000-2015, la tasa neta de deforestación en México fue de 121 mil hectáreas al año (Secretaría de Medio Ambiente y Recursos Naturales [SEMARNAT], 2016). Los bosques juegan un papel primordial en la regulación de las funciones del ciclo del agua, actuando como reguladores, limpiadores y proveedores del recurso hídrico; por ello es fundamental mantener los ecosistemas saludables, pues son éstos quienes se encargarán de mejorar la seguridad hídrica, por medio del equilibrio del ciclo del agua (Programa Mundial de las Naciones Unidas de Evaluación de los Recursos Hídricos [WWAP], 2018).

Michoacán es un estado considerado forestal, el cual presenta problemas de deterioro de sus bosques, similares al resto del país: presencia de deforestación, cambio del uso de suelo de forestal a agrícola - para el cultivo de aguacate principalmente-, aprovechamientos ilegales, incendios forestales, plagas y enfermedades, poco éxito en las reforestaciones, manejo forestal inadecuado, entre otros. Todo ello requiere de la implementación de planes regionales de desarrollo forestal, con la participación de todos los sectores, que contribuyan a mejorar el medio ambiente y la calidad de vida de la población que habita y cuenta con los bosques como su medio de vida (Ávila García et al., 2014; Comisión Forestal del Estado de Michoacán [COFOM], 2007; Gómez Durán, 2020).

Dicho estado se encuentra entre los primeros lugares del país con mayor velocidad de degradación de la vegetación natural (1,16 \%) en el periodo 2002 al 2011 (SEMARNAT, 2016). Asimismo, la superficie del cultivo de aguacate se incrementó en un $75 \%$, entre 1980 y 2012, con incidencia importante en la deforestación, estimada en, al menos, 690 hectáreas al año en el estado (Chávez-León et al., 2012).

Ante la problemática en torno a los bosques se han desarrollado políticas públicas por medio de programas, con la participación del sector público, privado, organizaciones sociales, etc.; sin embargo, la degradación persiste y las comunidades forestales continúan en la pobreza y marginación. De acuerdo con Santika et al., (2019), la evidencia de los impactos de los programas forestales, tanto en la conservación de los bosques como en el alivio de la pobreza es escasa. En la región del lago de Pátzcuaro, de acuerdo con Ávila García et al. (2014), existe una robusta presencia de programas institucionales para el desarrollo forestal, sin embargo, su impacto es mínimo o poco perceptible, al no contar con una estrategia que atienda de forma focalizada e integral la problemática silvícola de la región, articulándola con la pobreza, tala clandestina y la presencia del crimen organizado que ha hecho de los bosques una zona peligrosa y de alto riesgo para la población. Tales factores impactan negativamente en la capacidad de las comunidades forestales para construir visiones de largo plazo en torno a sus recursos naturales de uso común, ya que las presiones externas son determinantes en el deterioro del bosque. La percepción de la población local sobre dichos programas cobra relevancia, dado que a ellos van dirigidos y son quienes viven las consecuencias de los impactos; sin embargo, los avances en el estudio de las percepciones aún son puntuales y su número es escaso, por lo que, además de impulsar este tipo de investigación, es fundamental considerar que las percepciones no se generan de manera aislada. Siempre deben vincularse al contexto micro y macrosocial, relacionando la perspectiva histórica, económica y ambiental para, además, evitar considerarlas únicamente como opiniones (Cruz Angón et al., 2013). 
En este contexto se llevó a cabo una investigación exploratoria de corte etnográfico sobre percepciones de actores locales acerca del estado de conservación de sus bosques en la microcuenca de Ichupio, ubicada en la región del lago de Pátzcuaro, dicha zona corresponde a la región purépecha de Michoacán. Con este estudio se pretende identificar cómo la población de la región se relaciona con los bosques y percibe los efectos de las acciones de conservación de suelo y agua, realizadas en el marco del Programa para la Recuperación Ambiental de la Cuenca del Lago de Pátzcuaro (Praclp), llevado a cabo en la zona, con el objetivo de generar recomendaciones para el diseño y puesta en práctica de acciones más asertivas en cuanto a las necesidades y demandas locales, a fin de que puedan realmente cumplir con su mandato de recuperar y conservar la base de recursos naturales.

Se optó por desarrollar la investigación a partir de las percepciones ambientales, definidas como un "proceso cognitivo de la conciencia que consiste en el reconocimiento, interpretación y significación, para elaborar juicios en torno a sensaciones obtenidas del ambiente físico y social, en el que intervienen procesos como el aprendizaje, la memoria y la simbolización" (Vargas Melgarejo, 1994, p. 48). Por ser un concepto contextualizado en términos tanto espaciales como temporales, se arguye que la percepción de los actores sociales de la microcuenca Ichupio determinará cómo dichos individuos desplegarán estrategias para relacionarse con sus bosques, actuando o no de manera activa en los procesos de conservación, ya sean impulsados desde lo local o realizados a través de programas gubernamentales. En estos términos, comprender cómo las personas perciben y se comportan con relación al manejo de los bosques se vuelve un insumo crucial tanto para la academia como para quienes toman decisiones (Tarannum et al., 2018).

La selección del caso de estudio corresponde al interés de recuperar el sentir de los actores locales de la microcuenca respecto a sus bosques y al impacto de las acciones de conservación de suelo y agua del Praclp, el cual está integrado por una serie de proyectos para atender problemáticas específicas, entre ellas la degradación de los bosques y suelos, estableciendo vegetación a través de reforestaciones de pino, barreras vivas con pasto vetiver en los taludes de las cárcavas y construcción de presas para el control de cárcavas. La microcuenca Ichupio constituye un caso particularmente interesante para analizar el ejercicio de sondear las percepciones ambientales de actores locales respecto a la conservación de los bosques, dado que es una zona boscosa, con la presencia de pino y encino en la parte alta, en la media hay bosque alterado y cárcavas, mientras que en la porción baja una pequeña área agrícola; a la salida de la microcuenca se ubica el lago de Pátzcuaro. Por las características físicas del sitio, al remover la vegetación se generan fuertes problemas de erosión, pérdida de la fertilidad del suelo y producción de sedimentos que son depositados en el lago de Pátzcuaro, por esta razón la conservación de dicho bosque cobra extremada relevancia.

Al acercarse a las percepciones sociales de actores locales de la microcuenca Ichupio, se tiene la intención de brindar elementos para contestar a las siguientes interrogantes: ¿Cómo los actores locales perciben su acceso y uso del bosque?, ¿qué apreciación tiene la población acerca del resultado de las acciones de conservación de suelo y agua llevados a cabo por el Programa para la recuperación ambiental de la cuenca del lago de Pátzcuaro?, y ¿cuáles estrategias deberían contemplar las acciones, para que puedan responder a las demandas locales y así tener un mayor impacto positivo en la conservación ambiental? Para ello, se estructuró la contribución de la siguiente manera: primero, se realiza un acercamiento al marco conceptual de las percepciones sociales. Enseguida se despliega la metodología y la zona de estudio. Posteriormente, se presentan los resultados encontrados y, finalmente, se plantean conclusiones. Se considera que los hallazgos que aporta esta investigación reflejan lo que acontece en numerosas comunidades boscosas del país, en donde, pese a los recursos invertidos en propuestas de recuperación ambiental, perdura el proceso de deterioro y pérdida de la biodiversidad, y por ello es ineludible repensar su diseño y estrategias de implementación y seguimiento.

\section{Acercamiento a las percepciones ambientales}

Uno de los pioneros en el estudio de las percepciones fue Tuan (1974), quien arguye que la percepción es el resultado de la sensibilidad a los estímulos externos, en donde se registra información de la realidad de manera parcial, generando una visión particular del mundo, que se vuelve un insumo importante para el desarrollo del comportamiento individual y social. Tuan, acuña el concepto de "topophilia", el cual se refiere a la articulación subjetiva entre las personas y su espacio y que debe ser analizado desde los ámbitos temporal (histórico), espacial (geográfico) y cultural (antropológico) (Fernández Moreno, 2015). 
Según Bertoni y López (2010), las percepciones ambientales son el resultado de la actividad cognitiva, cultural y simbólica de los grupos sociales y los patrones de interacción sociedad-naturaleza, que sustentan y orientan el manejo de los recursos naturales, son realizados desde dichos referentes. Los autores añaden que las percepciones son formuladas a través de juicios que involucran evaluaciones de lo percibido, construidas desde la subjetividad, dando como resultado lecturas a favor o en contra de determinados aspectos del ambiente, las cuales determinarán actitudes positivas o negativas hacia la conservación. Por ello la relevancia del estudio de percepciones para la conservación de los bosques, al brindar un marco analítico que permite develar las intenciones detrás del actuar en la realidad. Hay una amplia gama de estudios enfocados en las percepciones sociales relacionadas con el acceso y manejo de los recursos naturales, ya sea desde la psicología ambiental, la cual se centra en los individuos, o desde la geografía, considerando los grupos sociales y cómo los factores culturales juegan un rol significativo en cada sociedad. En el campo de la antropología el estudio de las percepciones viene ganando mucha fuerza, por brindar información relevante desde el referente empírico, acerca de los valores, las creencias y actitudes que se crean y recrean como una estrategia de apropiación de la realidad por los sujetos sociales, lo que se constituye en una herramienta importante para la toma de decisiones (Chanca Flores et al., 2020; Fernández Moreno, 2015).

La antropología mexicana ha aportado significativamente al estudio de las percepciones, de tal suerte que los trabajos de Arizpe et al. (1993), Vargas Melgarejo (1994) y, Lazos Chavero y Paré Ouellet (2000), son sólidos referentes en la materia. Para Arizpe et al. (1993), dos elementos confluyen en la conformación de las percepciones: uno proveniente de la experiencia individual directa con el ambiente y, el otro, que se deriva de la interacción social, de los medios de comunicación, de la ciencia, etc., por lo tanto, considerado indirecto. Vargas Melgarejo (1994) asume que la percepción está enmarcada en el límite entre biología y cultura, dado la capacidad biológica y de producción de símbolos de los seres humanos. Por otro lado, Lazos Chavero y Paré Ouellet (2000) aseveran que las percepciones guían los procesos subjetivos que influyen en la toma de decisiones. Dichos autores coinciden en el hecho de que las percepciones producen significaciones y que éstas son atributos culturales, derivados de interacciones sociales, por lo tanto, no hay una realidad objetiva, sino diferentes interpretaciones de los procesos de degradación y deforestación, en función de las vivencias del grupo social (Durand, 2008).

La percepción de los grupos sociales locales acerca del uso sostenible de los bosques es esencial para su manejo efectivo, dado que la forma como las personas perciben y valoran su entorno condiciona su proceso de toma de decisiones sobre el ambiente, de tal suerte que conocer las percepciones ambientales de pobladores locales brinda información relevante para hacer más eficiente las políticas de conservación. Asimismo, la conservación de la biodiversidad no es un asunto exclusivamente de carácter científico o técnico, sino una condición para garantizar la supervivencia material y cultural de las poblaciones poseedoras de los recursos, por ello, es ineludible indagar sobre las diferentes percepciones locales respecto de qué, por qué, cómo y cuándo se debe conservar (Bertoni y López, 2010; Fernández Moreno, 2015). Al conocer la percepción de pobladores de la microcuenca Ichupio sobre la conservación de sus bosques, se trata de comprender la subjetividad social, determinando las formas en que la población se apropia de su entorno natural y, por lo tanto, sus intereses y compromisos con su conservación y manejo sostenible.

La organización juega un papel muy importante en este contexto; la organización comunitaria de los pueblos y comunidades indígenas p'urhépecha data desde los tiempos prehispánicos. Ésta se expresa actualmente de diferentes formas, pese a una constante presión externa proveniente principalmente del mundo mestizo. Una de las formas más significativas es la que se asocia a los usos y costumbres tradicionales, determinando la dinámica de los actores sociales y su interacción en las acciones comunitarias que giran en torno a las temporadas productivas y a las festividades patronales que a lo largo del año se realizan en cada comunidad, pueblo y cabecera municipal (Amézcua Luna y Sánchez Díaz, 2015).

Precisamente, mediante esta forma de organización han surgido comunidades que luchan en la región para recuperar sus bosques, como es el caso de Cherán, quienes se organizaron en defensa de su bosque y territorio frente a grupos delictivos que habían devastado el bosque para venderlo y establecer huertas de aguacate (Suarez, 2019). Otras comunidades se han organizado y realizan manejo forestal comunitario que da empleo a la comunidad e incide en la conservación del bosque como es el caso de Nuevo San Juan Paragaricutiro; las especies de pino de esta zona denominadas Pinus michocanaca y P. Pseudostrobus, tienen gran demanda en el mercado nacional por ser más flexible y consistente que las importadas de China y Chile (Del Castillo, 2020).

En Ichupio también se rigen por usos y costumbres. El nombramiento de sus autoridades agrarias se realiza cada tres años por asamblea comunitaria, quienes representan a la población en lo relacionado con la tierra. 
Por otra parte, cada año se nombra a las autoridades comunitarias; a la autoridad principal se la denomina "encargado del orden"; ambas autoridades juegan un papel importante en lo relacionado con la tenencia de la tierra y el aprovechamiento del bosque, pues en asamblea comunitaria se toman dichos acuerdos.

\section{Metodología}

Para conocer el acceso y uso del bosque por parte de los habitantes de Ichupio, así como la percepción que tiene la población acerca del impacto de las acciones de conservación de suelos llevado a cabo en la microcuenca, se partió de una aproximación etnográfica y el empleo de herramientas de corte cualitativo, con la realización de entrevistas semiestructuradas y observación no participante.

Según Pérez Andrés (2002), mientras las técnicas cuantitativas investigan los hechos, las cualitativas buscan entender los procesos de producción de los hechos, es decir, revelan el origen y la formación de los sentidos detrás de su contenido manifiesto. Los seres humanos se relacionan con la naturaleza y, mediante un proceso de simbolización, interpretan dicha relación por medio de la abstracción. La investigación cualitativa propone la elucidación de las unidades de sentido de esta interacción, evidenciando la subjetividad, la cual, a su vez, está condicionada por los contextos social, cultural e histórico particular en el cual las personas se desarrollan. La entrevista, como herramienta de investigación cualitativa, posibilita generar información sobre las percepciones respecto a determinados temas relacionados con la vida social, en este caso, la relación con el bosque y el impacto de las acciones de conservación. El rol del investigador es clave en la entrevista, dado que, para la obtención de información, debe establecer una relación directa, cordial y empática con los sujetos de la investigación, a fin de generar la confianza que posibilita un diálogo fluido (Zerpa de Kirby, 2016). Las entrevistas semiestructuradas parten de preguntas planeadas, que pueden ajustarse a los entrevistados, posibilita aclarar términos, ambigüedades y reducir formalismos (Díaz-Bravo et al., 2013); debido a su flexibilidad permite obtener información más profunda y detallada que las entrevistas estructuradas.

Las categorías de análisis y variables fueron definidas a fin de permitir una articulación entre las percepciones locales y propuestas de lineamientos generales a considerarse en proyectos de recuperación ambiental, con el objetivo de construir una reflexión acerca de la necesidad de reorientar procesos de conservación en el ámbito local. El diseño de las entrevistas contempló la definición de variables relacionadas con la identificación de las personas informantes, actividades principales, acceso y usos del bosque, conocimiento de programas y acciones de conservación llevados a cabo en la zona de estudio, participación en las acciones y valoración de su impacto. Se aplicaron doce entrevistas semiestructuradas, a cuatro mujeres y ocho a hombres, a informantes claves de la localidad de Ichupio, mayores de edad y con un mínimo 10 años de residencia en la zona.

Aunado a las entrevistas se realizó la observación no participante, la cual es una técnica de recolección de datos que consiste en prestar atención mediante el empleo de los sentidos a un fenómeno y/o grupo de individuos de interés de estudio, con la finalidad de obtener información y registrarla de manera sistemática para su posterior análisis. Los ejes de la observación fueron el estado del bosque y las técnicas de conservación de agua y suelo llevadas a cabo en la zona. Asimismo, la observación permitió cruzar datos con las entrevistas y reflexionar acerca de las respuestas, contextualizándolas con lo observado.

Para el trabajo de campo se contó con el permiso de la autoridad local, conocida localmente como "encargado del orden", así como con el consentimiento previo e informado de cada una de las personas entrevistadas. Se realizaron dos estancias de trabajo de campo: una exploratoria, a través de recorridos de campo con guías locales para visitar la zona boscosa y el lago de Pátzcuaro, en el límite con la localidad de Ichupio; y una extensiva, para la realización de las entrevistas. La observación no participante fue una técnica empleada en ambas visitas a la zona.

\section{Zona de estudio}

La microcuenca Ichupio correspondiente a la cuenca del lago Pátzcuaro, se sitúa en la localidad de Ichupio, en el municipio de Tzintzuntzan, estado de Michoacán. Hidrológicamente pertenece a la cuenca del lago Pátzcuaro y ésta, a su vez, a la Región Hidrológica 12 Lerma-Santiago. Cuenta con una superficie de 101,95 ha, se encuentra entre los 2.040 y $2.520 \mathrm{~m} \mathrm{~s}$. n. m, con una pendiente media de $29,75 \%$. En la Figura 1 se muestra su ubicación. 


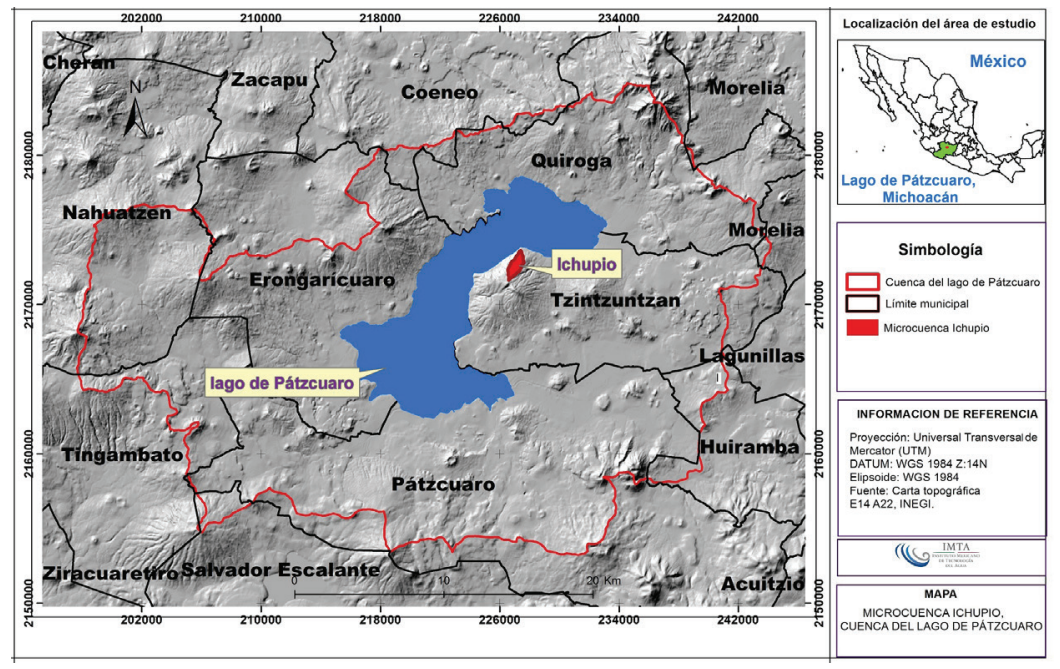

Figura 1. Localización de la microcuenca Ichupio, cuenca del lago de Pátzcuaro, Michoacán.

Figure 1. Location of the Ichupio micro-basin, lake Pátzcuaro basin, Michoacán.

Por las características de relieve y suelo, la vocación principal de la microcuenca es forestal, corresponde con el uso de suelo actual, pues alrededor de tres cuartas partes (72,50\%) es de uso forestal; constituido de bosque de pino, encino y madroño; las principales especies en la región son Pinus teocote, P. michoacana, P. montezumae, P. patula, P. pseudostrobus, Quercus rugosa, Q. laurina y Arbutus xalapensis (Instituto Mexicano de Tecnología del Agua [IMTA] y Fundación Gonzalo Río Arronte, 2004).

La precipitación promedio se aproxima a los $800 \mathrm{~mm}$ anuales; la época lluviosa corresponde a los meses de junio a septiembre, en este período incrementa el azolve del lago de Pátzcuaro ocasionado por el arrastre de sedimentos de las laderas desprovistas de vegetación. En la microcuenca existen dos unidades de suelo: el acrisol, caracterizado por la acumulación de arcilla en el subsuelo, ácido y pobre en nutrientes, con rendimientos muy bajos en la agricultura y moderadamente susceptibles a la erosión, por ello, el uso más adecuado para su conservación es el forestal. Este suelo cubre 95,5\% de la superficie total de la microcuenca, el resto (4,5 \%) corresponde a luvisol, se identifica por sus tonos rojos, amarillentos o pardos, se destinan regularmente a la agricultura, con rendimientos moderados (INEGI, 2014).

Con relación a la demografía, Ichupio es una localidad indígena de 272 habitantes, de los cuales 143 son mujeres y 129 hombres, quienes habitan 71 viviendas. El grado de marginación es alto, 4,41 \% de la población mayor a 15 años no sabe leer ni escribir, solo el 50,70 \% de las viviendas habitadas disponen de agua entubada y se abastecen de la red pública, $42,25 \%$ de viviendas particulares habitadas no disponen de drenaje, por lo que utilizan baños secos (INEGI, 2020).

El grupo étnico p'urhépecha se ha asentado en una región con abundantes recursos naturales, entre ellos bosques, zonas lacustres y suelos ricos, dando cabida a la conformación histórica de un patrimonio biocultural que brinda al pueblo el sentido de pertenencia e identidad. Si bien las transformaciones de la región han sido paulatinas, se ha venido incrementando a partir de la segunda mitad del siglo xx, con un proceso de explotación forestal con consecuencias devastadoras para la biorregión. Ello, aunado a la migración, tanto de p'urhépechas hacia los Estados Unidos, en búsqueda de mejores oportunidades de vida, como de mestizos hacia la región de la cuenca del lago de Pátzcuaro, ha impactado en la generación de nuevos hábitos de consumo y pérdida de usos y costumbres tradicionales, con consecuencias negativas no solo en el tejido social comunitario tradicional sino en el ambiente. Estos cambios han propiciado el creciente abandono de actividades de conservación y protección de los recursos naturales o la indiferencia a su depredación (Amézcua Luna y Sánchez Díaz, 2015).

En la actualidad el bosque ya no es el eje de la vida comunitaria y la población debe conjugar una serie de labores para obtener sus medios de vida, entre ellas la agricultura, pesca y elaboración de artesanías, aunado a trabajos de albañilería, comercio o servicios. La pesca, otrora la principal fuente de ingresos de la población, se ha reducido drásticamente debido a una serie de factores, entre ellos, la contaminación del lago y la disminución de la especie endémica de mayor precio en el mercado (pescado blanco), por la presencia de especies exóticas (carpa y tilapia). La artesanía consiste en la elaboración de una serie de productos en talleres familiares, como muebles, cestos, tapetes, figuras de animales, etc., los cuales se venden en Tzintzuntzan, Pátzcuaro, 
ferias locales y en diferentes regiones del país. Debido al proceso de deterioro ambiental, con la merma de los recursos naturales, la diversificación productiva se incrementa, dando cabida inclusive al trabajo asalariado en el mercado regional o internacional, principalmente en los EUA. Estos cambios en la vida productiva y económica afectan los estilos de organización del trabajo familiar a la par que promueven un alejamiento de conocimientos y experiencias relacionadas con los recursos naturales, que desde siempre estuvieron presentes como integrantes del acervo patrimonial colectivo (Argueta y Castilleja, 2008).

A pesar de que en la microcuenca únicamente se cuenta con dos hectáreas de área agrícola, es una actividad importante y las demás actividades se desarrollan respetando los tiempos dedicados a la agricultura. El papel de la mujer en las labores agrícolas es sustancial, debido a la migración de los hombres hacia Estados Unidos u otras partes del país. La migración expresa la carencia de trabajo para la población y eso lleva implícito una creciente desvinculación con el trabajo de la tierra, inclusive terrenos cultivados han sido abandonados y la población ya no es autosuficiente en la producción de maíz (IMTA, 2011).

Debido a los fuertes procesos de erosión y el consecuente azolvamiento del lago de Pátzcuaro el valor turístico de la región, se ha visto comprometido con un impacto directo en la economía regional. Durante muchos años se han implementado diversos programas de restauración y conservación de los bosques en la cuenca, a través de fuentes de financiamiento internacional, nacional y regional, llevados a cabo por el sector público, privado, organizaciones sociales, etc. Uno de los programas de gran aliento fue el de Recuperación Ambiental de la Cuenca del Lago de Pátzcuaro, con una duración de 14 años, el cual comenzó en el año 2003 y concluyó en 2017, cuyo objetivo fue revertir la problemática ambiental de la cuenca con una serie de actividades, entre ellas la reforestación, el impulso de tecnologías alternativas para el acceso al agua, el desarrollo de saneamiento ecológico y la educación ambiental (García Villanueva, 2009). En la microcuenca Ichupio el referido programa concentró sus acciones en la promoción de la reforestación, construcción de barreras vivas y de presas para el control de cárcavas.

\section{Resultados y Discusión}

En este apartado se analiza el acceso y uso del bosque por los habitantes de Ichupio, así como la percepción que tiene la población acerca del impacto de las acciones de conservación de suelo y agua y, a partir de dichos insumos, plantear algunas estrategias que deberían contemplar las gestiones de recuperación de bosques, que permitan responder a las demandas locales y así tener un mayor impacto positivo en la conservación ambiental.

Según Argueta y Castilleja (2008), las percepciones, derivadas de prácticas de observación y análisis de la naturaleza, están vinculadas al cuerpo de creencias del grupo social y posibilitan su reproducción como colectivo y no solamente como individuos. Por lo cual, comprender los procesos de acceso, uso y valoración de recursos en el ámbito local permite un acercamiento a las concepciones locales, lo que da cabida a la posibilidad de diálogo entre saberes, con el fin último de lograr la conservación de los bosques. Para algunos pobladores mayores de Ichupio, la conservación del bosque se relaciona con la herencia que recibieron de sus ancestros y que, a su vez, ellos podrán heredar a sus hijos y nietos. Forma parte de su identidad y, junto con el lago, influye en las actividades productivas locales. Asimismo, manifestaron que anteriormente los pobladores de la cuenca realizaban reforestaciones a través de faenas. Para otros, la percepción incluye el uso y manejo del bosque, en esta relación con el componente social, consideran que el aprovechamiento del bosque es para subsistencia.

\subsection{Acceso y uso del bosque}

En la microcuenca Ichupio predomina el uso de suelo forestal con bosque de pino y encino, en menor cantidad existen otras especies nativas como el madroño. El bosque se aprovecha para leña principalmente Quercus sp. (encino) y elaboración de artesanías Arbutus xalapensis (madroño). Adicionalmente, se obtienen productos alimenticios, como hongos, nopal en época de lluvia, tierra para la elaboración de trastes de barro y flores para algunas festividades; por ejemplo, la orquídea que se utiliza en las ofrendas del día de muertos en diferentes localidades de la región, incluido Ichupio. El aprovechamiento del bosque por Ichupio es para autoconsumo, principalmente; no se observaron extracciones de madera o aprovechamiento a gran escala, sin embargo, dentro del bosque se encuentran áreas desprovistas de vegetación, bosque alterado y erosión en cárcavas.

Localmente la población tiene áreas específicas para los diferentes usos del suelo; bosque en la ladera y agricultura en la parte baja, lo que de alguna forma ha repercutido en la conservación del bosque pese a la 
problemática en torno al mismo. La tala ilegal, si bien está presente en la cuenca, en Ichupio no representa un grave problema. En la localidad el área de bosque y agricultura están bien definidas, sin embargo, de acuerdo con los habitantes, principalmente de la tercera edad, el bosque es menos denso y dan testimonio de la abundancia de pino y encino, expresan que era tan espeso el bosque que les resultaba difícil caminar en él, dado que disponían de pinos de gran altura y grosor, pero todo se redujo por la gran extracción de madera y la producción de carbón (García Villanueva, 2009). Dicho resultado coincide con el encontrado por Peralta-Rivero et al., (2016) en su investigación realizada en la huasteca potosina, en donde las personas entrevistadas aseguran que en el pasado había abundancia de árboles frondosos en comparación con la actualidad, lo que indicaba el buen estado de conservación de los bosques y su gradual degradación. Otro punto abordado por los entrevistados fue la existencia de áreas degradadas dentro del bosque, con la presencia de erosión en cárcavas, como consecuencia, el suelo se vuelve susceptible a la erosión hídrica y eólica, los escurrimientos arrastran sedimentos que desembocan en el lago de Pátzcuaro y generan azolve, lo que influye directamente en las actividades productivas: pesca y agricultura de la población de Ichupio.

No existe manejo forestal comunitario - lo que limita el aprovechamiento del potencial forestal—, que conlleve a la conservación del bosque a largo plazo para el desarrollo y beneficio económico de la población. Ello es un nudo relevante para la conservación y manejo sostenible de los ecosistemas, pero debería plantearse como el horizonte deseable, y para ello habría que sortear varios retos, entre ellos: ambientales, relacionados con el mantenimiento del hábitat y la diversidad biológica; económicos, concernientes a generar ingresos competitivos en comparación con otras actividades; sociales, relativos a mantener la organización y cohesión en torno al cumplimiento de las reglas establecidas colectivamente y, finalmente, pero no menos importante, los tecnológicos, relacionados con las operaciones de manejo y procesos de planeación (Aguirre-Calderón, 2015). El manejo del bosque debe contribuir a mejorar la resiliencia, la capacidad de aprendizaje y la adaptabilidad de las comunidades, al tiempo que ayuda a reducir las amenazas, los riesgos y las vulnerabilidades (Torres-Rojo et al., 2016). La destrucción o conservación del bosque depende de la capacidad de sus propietarios (incluido el Estado) para planificar y reglamentar su gestión, aplicar sanciones a quienes contravengan los criterios de uso y producir ingresos económicos a partir de su aprovechamiento sustentable para evitar el cambio de uso de suelo hacia usos no forestales (Zúñiga y Deschamps, 2013).

Existe un problema añejo, relacionado con el acceso al bosque y la tenencia de la tierra, entre el municipio de Tzintzuntzan y la localidad de Ichupio, lo cual contribuye a la degradación de los recursos, debido a la incertidumbre y el potencial conflicto entre actores sociales. Ello, en virtud de que el entonces presidente de la República, Gral. Lázaro Cárdenas (1934-1940), autorizó el aprovechamiento para leña, del bosque de Ichupio, por la cabecera municipal de Tzintzuntzan. Actualmente, existen diferencias entre ambas localidades por la tenencia de la tierra y uso del bosque. Ichupio argumenta que es el dueño legal del bosque y aunque Tzintzuntzan realiza aprovechamiento, no participa en su conservación, como lo señalan varios entrevistados:

Cárdenas autorizó que aprovecharan nuestro bosque para leña; ahora, hasta dicen que son los dueños (Tzintzuntzan), pero nosotros tenemos documentos que nos acreditan como propietarios legales (persona mayor de la localidad de Ichupio).

Nosotros participamos y cuidamos el bosque cuando hay incendio, ¿pero de qué sirve?, si los de Tzintzuntzan suben al cerro a cortar leña para venderla y ellos nunca siembran o cuidan el bosque (mujer adulta, ama de casa).

Inclusive, habitantes de Ichupio señalan que algunos de sus terrenos se encuentran en el área que Tzintzuntzan pretende que se les reconozca (Sentencia pronunciada en el expediente 423/02). Ellos expresan que la reforestación del bosque tiene entre sus beneficios que éste pueda ser declarado área natural protegida, a fin de consolidar su conservación y asegurar la pertenencia de la tierra a Ichupio. Otra situación relacionada con la tenencia de la tierra y la pérdida de bosques es la venta de terrenos. Como consecuencia de la crisis de la agricultura y la pobreza creciente, la tierra se vende a foráneos y se trasforma en huertas de aguacate (IMTA, 2011) o en casas de descanso de fin de semana donde laboran habitantes de Ichupio en su mantenimiento.

Adicionalmente, por tradición, en la comunidad se deben cumplir comisiones durante las fiestas patronales y los funerales, lo que representa un costo elevado para los habitantes, al grado de incluso vender sus propiedades para solventar esta responsabilidad. La pobreza, el compromiso con las festividades, la falta de oportunidades de trabajo, entre otras complejas problemáticas, incluyendo la migración, se articulan para orillar a los 
dueños de los bosques, la venta de sus tierras, por lo cual la base de recursos naturales se transforma en moneda de cambio, lo que trae como consecuencia la reducción de la biodiversidad forestal y, a la larga, el ciclo vicioso del empobrecimiento de la población y la pérdida de sus medios de vida. Esta situación no es exclusiva de la microcuenca Ichupio, sino es compartida a lo largo y ancho de las comunidades boscosas del país. De hecho, en la región Huasteca, Peralta-Rivero et al. (2016) documentaron el incremento de la deforestación para la diversificación de las actividades productivas y económicas, argumentando que estos procesos son complejos y requieren de un enfoque multidisciplinario.

\subsection{Percepción del impacto de las acciones de conservación de suelo y agua llevadas a cabo en la micro- cuenca Ichupio}

Ichupio es una localidad donde se ha realizado continuamente reforestación, a través de diferentes programas o proyectos, con amplia participación de la Delegación Forestal VII Pátzcuaro de la Cofom. Los programas de conservación han contribuido parcialmente en la conservación del bosque y el lago, así como en dar empleo temporal. Sin embargo, de acuerdo con las opiniones vertidas por la población, se han tomado decisiones de manera unilateral con el objetivo inicial de mejorar las condiciones de vida de los habitantes, sin embargo, los resultados han afectado negativamente a algunas localidades dentro de la misma cuenca, en especial a quienes habitan en la ribera del lago y se dedican a las actividades productivas primarias, como es Ichupio. Un ejemplo de ello fue la introducción de especies exóticas (tilapia) al lago, lo que afectó a la población de peces originarios "pez blanco", el cual tiene mayor valor comercial, llegando al punto que su población actual es mínima, lo que afecta el ingreso de los pescadores y su dieta. Un testimonio al respecto:

El gobierno tiene la culpa de que ahora haya muy pocas especies originarias del lago, porque ellos introdujeron especies que afectaron gravemente a las especies nativas, ya que la tilapia que llegó comía los huevos del pescado blanco (pescadora y maestra jubilada).

Este tipo de resultados inesperados con el desarrollo de programas en la zona y con un impacto negativo en las condiciones de vida local, ha dejado como saldo un desgaste social muy importante y una desconfianza arraigada hacia programas e intervenciones del gobierno e incluso de instituciones académicas y de organizaciones de la sociedad civil (Vargas Velásquez, 2014). Aunado a ello, Millán Malo (2005) menciona que las estrategias de algunos programas fomentaron una relación paternalista y benefactora entre el gobierno y la población, y en otras ocasiones se prometían cosas que no se cumplían, lo que trajo como consecuencia que los canales de participación entre las comunidades, las organizaciones de la sociedad civil y el gobierno estén deteriorados y en algunos casos rotos.

Un programa que tuvo presencia de largo aliento en la zona fue el Programa para la Recuperación Ambiental de la Cuenca del Lago de Pátzcuaro, cuya meta fue combatir la problemática ambiental, con objetivos y actividades de diferente índole y con una permanencia temporal de catorce años (2003-2017). En la microcuenca Ichupio se realizaron acciones de reforestación y de regulación de la erosión del suelo, mediante la construcción de presas para el control de cárcavas (García Villanueva, 2009).

En dicho programa no han existido estrategias de participación efectiva y acceso a la toma de decisiones por parte de actores sociales locales, ni canales de fortalecimiento de capacidades y de organización, sino que más bien se ha considerado la participación social en la etapa de ejecución de las actividades, bajo el esquema de empleo temporal, a fin de fortalecer la economía local. Esta estrategia de contratación de mano de obra local para realizar actividades puntuales es compartida, entre otros, por el programa ProÁrbol, como una opción atractiva para obtener recursos económicos por parte de los beneficiaros, sin embargo, es un beneficio temporal, que no impacta de manera duradera en la economía local y tampoco en los procesos organizativos (Jiménez Pérez, 2009).

Además de que la población local no haya sido tomada en cuenta para la planeación de las acciones, hubo fallas de carácter logístico, las cuales han influenciado negativamente, no solo en el logro de los objetivos, sino en la confianza de la población hacia el gobierno. Un ejemplo de ello es la estrategia inadecuada de reforestación, percibida por los habitantes locales, quienes aseveran que la planta para reforestar se entregó una vez avanzado el periodo de lluvias, lo que repercute en el desarrollo de la vegetación. Asimismo, argumentan que el retraso en la entrega de las plantas no es una debilidad exclusiva de este proyecto, sino es compartida por varias estrategias, en donde los tiempos burocráticos administrativos no suelen coincidir con los ambientales, trayendo como consecuencia que el porcentaje de éxito de las reforestaciones no sea el esperado. 
Otro argumento presentado en contra del éxito de la reforestación es el trasplante de los arbolitos en áreas muy degradadas, en donde no existe suelo y las raíces no pueden penetrar, repercutiendo en la sobrevivencia y desarrollo de la planta. Las personas entrevistadas mencionaron que se debería establecer vegetación en el sedimento retenido por las presas, es decir, identificaron la oportunidad de reforestar donde ya se ha formado suelo, puesto que en las reforestaciones la falta de suelo limita la sobrevivencia de la vegetación. Ello revela el conocimiento sobre la dinámica ambiental del territorio por parte de la población, el cual podría ser utilizado con el fin de mejorar los resultados del proyecto, no solo por la experiencia empírica que encierra, sino también por el reconocimiento de los saberes locales, lo que, desde luego, actuaría en crear mayor confianza y reciprocidad entre instituciones y población local. A pesar de que los entrevistados conozcan sobre estrategias eficaces de reforestación y lo manifiesten en las entrevistas, dicho conocimiento no se refleja en su quehacer en el territorio, toda vez que, en los recorridos de campo, no se encontró establecimiento de vegetación por iniciativa propia en los sedimentos retenidos por las presas.

Asimismo, los habitantes locales proponen, con el objetivo de potenciar el impacto positivo de los programas, que exista mayor difusión, capacitación y que se incorpore a la población en los procesos de toma de decisiones, dado que ellos tienen conocimiento acerca de sus bosques, los cuales deberían ser retomados para el desarrollo de acciones más integrales como, por ejemplo, la obtención de productos forestales no maderables, como hongos. Asimismo, plantean que en las reforestaciones deberían tomarse en cuenta especies nativas de uso local para artesanías y leña, como el encino y el madroño, sin embargo, se privilegia el pino debido al periodo de germinación reducido en comparación con las otras especies.

Otro punto débil de las acciones, según percepciones locales, es la falta de seguimiento. Los promotores solamente contratan personal local para efectuar la reforestación y no regresan periódicamente a verificar el resultado y plantear acciones correctivas, en caso de ameritarlo. Tampoco la población local efectúa un proceso de evaluación y seguimiento de las actividades realizadas y, desde el punto de vista económico, la carencia de un seguimiento puntual de las acciones permite que las personas puedan beneficiarse de empleo temporal, repitiendo las actividades de reforestación en otros programas, en el mismo espacio. Esto revela que el nivel de relevancia dado a la conservación de los recursos naturales es inferior a sus intereses o necesidades económicas. Este punto es particularmente sensible para la recuperación de bosques, en donde los efectos de acciones de conservación se hacen notar en el mediano y largo plazo.

Los actores sociales locales señalan una diversidad de puntos débiles en las acciones de recuperación de bosques, entre ellos: la ausencia de la población local en los procesos de toma de decisiones; fallas de carácter logístico, con la entrega de plantas a destiempo; carencia de una visión más integral del bosque y falta de seguimiento. Por otro lado, también es cierto que valoran las actividades llevadas a cabo, de hecho, un porcentaje importante de las personas entrevistadas percibe que las acciones de conservación realizadas en la microcuenca Ichupio representan beneficios para la comunidad y el lago, relacionándolos principalmente con la reducción en la pérdida de suelo por las corrientes de agua y, a su vez, disminución de los sedimentos depositados en el lago.

Esta percepción social local positiva, en términos de recuperación ambiental, es avalada por mediciones de escurrimiento y sedimento a la salida de la microcuenca desde el año 2010 a la fecha, en donde se comprueba que ha disminuido el arrastre de sedimentos después de que se construyeron las presas y se ha establecido vegetación. Éste es un aporte de investigación que difícilmente se observa en otras cuencas del país intervenidas, debido a la carencia de una línea base y, como tal, es imposible saber si la percepción coincide con la realidad en términos de beneficios. Según Chanca Flores et al. (2020), la carencia de construcción de una línea base que permita medir con precisión su nivel de impacto, es compartido por una serie de programas a nivel latinoamericano, por lo cual no se cuenta con una evaluación precisa de los resultados de los programas en términos de cambios con relación a la situación original, antes de la ejecución de las acciones del programa. Además del beneficio ambiental, perciben la ayuda económica, al aseverar que el programa representó una relevante fuente de ingreso para sus familias, al participar del empleo temporal para la reforestación y construcción de presas.

Otro factor que hay que tomar en cuenta en las acciones de conservación es la tenencia de la tierra y su impacto en la exclusión de grupos sociales que no poseen terreno. En la microcuenca las acciones de conservación se realizaron en terrenos comunales y los comuneros son principalmente hombres, limitando la participación de las mujeres y jóvenes, reforzando la idea de que la responsabilidad de conservar recae en los hombres adultos y debilitando el sentido de pertenencia y corresponsabilidad hacia el territorio de los actores no involucrados en dichos procesos. Ello contribuye a que cada vez más los jóvenes se desvinculen del campo.

Asimismo, de manera general, los programas de conservación se fundamentan en las percepciones de quienes toman las decisiones y no de actores locales — dueños de los recursos—, situación que impacta nega- 
tivamente en su posibilidad de éxito; como ocurre en el nevado de Toluca, donde es probable que la población local no vea como propio el cuidado y conservación del bosque; entonces a mediano plazo, el deterioro continuará y el bosque desaparecerá (Endara Agramont y Herrera Tapia, 2016).

El estudio de percepciones sobre conservación en el ámbito local permite retomar los intereses y demandas de actores locales para el diseño de programas más incluyentes, encaminados a satisfacer los intereses de los poseedores de los bosques y ello podría potenciar el éxito de dichas iniciativas y así contribuir realmente a que se recuperen y se conserven los bosques. De hecho, en nuestro país existen casos de empoderamiento de comunidades forestales indígenas que, a través del arraigo a la tierra, el manejo y aprovechamiento de sus recursos naturales han logrado consolidar empresas comunitarias como los ejidos Amanalco, en el estado de México, Ixtlán de Juárez y San Pedro el Alto en Oaxaca, San Juan Nuevo en Michoacán o el ejido Caobas en Quintana Roo (Suarez, 2017), lo que les ha permito mejorar su situación económica y conservar los bosques.

La exclusión de las perspectivas, valores y creencias de la población local en el desarrollo e implementación de políticas de conservación no solo obstaculiza el proceso de apropiación del programa y compromiso con su éxito por actores locales; sino que puede llegar a generar conflictos entre las comunidades por el uso, manejo y conservación de sus recursos naturales, como ocurre en algunas áreas naturales protegidas en México; por ejemplo: el área natural protegida Archipiélago de Bosques y Selvas de Xalapa, Veracruz (Hensler y Merçon, 2020).

Asimismo, no hay que obviar el papel de la cultura en la construcción de las percepciones, toda vez que, si bien los sentidos siguen principios de reacción ante estímulos, constituyéndose una respuesta biológica, dicha respuesta integra un sistema más amplio que responde a normas y contextos sociales, culturales y económicos, motivo más que suficiente para retomar las percepciones de los grupos sociales locales pues, seguramente, serán diferenciadas de las de quienes diseñan las políticas. En la medida en que las percepciones dependen de las historias personales y se definen a partir de hábitos y vivencias, dado que su conformación está moldeada por la perspectiva desde la cual interactuamos con el mundo, percibimos exclusivamente lo que podemos leer de la realidad, en función de nuestras experiencias (Feria Zapata et al., 2019).

\subsection{Lineamientos a contemplarse en actividades de recuperación de bosques}

Los escasos resultados encontrados con la aplicación sistemática de programas de conservación de bosques, en la cuenca del lago de Pátzcuaro, no son una excepción en el país en términos de impactos de las políticas públicas en la recuperación ambiental, desafortunadamente hace parte de la regla. De hecho, Cotler et al. (2007) proponen como una causa importante de dichos resultados, la lógica que rige los programas, la cual se centra en el beneficio económico para la población y el interés político del gobierno de turno. Un argumento que valida los planteamientos de Cotler son los escasos indicadores sobre el efecto de los programas a mediano y largo plazo en la estabilidad de los bosques, pues los indicadores se concentran en la acción en sí y no en los resultados esperados.

Asimismo, y abonando a los argumentos anteriores, está la absoluta carencia de coordinación intersectorial, con políticas agrícolas contradictorias a los intereses de conservación de la biodiversidad en el país, como son la ganaderización y el impulso a los monocultivos con elevada dependencia de agroquímicos; prácticas que, lamentablemente, han acentuado la problemática de degradación y pérdida de los bosques. Aunado a ello existe la falta de continuidad sexenal de las políticas públicas, de tal suerte que, por más bien intencionado que sea un programa, el horizonte temporal de su aplicación no permite un impacto decisivo en la fortaleza del capital natural, debido a la falta de concordancia entre los tiempos requeridos por la naturaleza para sus procesos y los político-administrativos. Ello conlleva a una contradicción latente entre las instituciones, las políticas y las acciones concretas que se realizan en los territorios.

A partir de este breve recuento relacionado con el porqué los programas no tienen el impacto deseado en la conservación de los ecosistemas, empezamos a proponer lineamientos que pudieran contribuir a revertir esta tendencia. El primero de ellos es la necesidad del entendimiento del problema y sus causas, desde la perspectiva de los actores locales, por ello, conocer sus percepciones es un paso primordial para empezar a delinear nuevos rumbos para la recuperación de los bosques. A partir de la construcción del problema a nivel local, en donde los actores sociales perciben la afectación en sus vidas y relacionan sus causas, se incluye el problema en la agenda gubernamental y se diseñan programas para atacar sus causas, respondiendo a intereses y expectativas de las poblaciones que dependen de su capital natural. Ello se constituiría en un aliciente para que se comprometan con el seguimiento de las acciones, que es un nudo importante para el éxito de los programas. 
Asimismo, ello implicaría la realización de diagnósticos de las distintas realidades y problemáticas derivadas de la articulación de grupos sociales-bosques, implicando una territorialización de los programas. Un enfoque territorial aportaría a una comprensión más integral de las variadas situaciones del patrimonio natural de los pueblos, evidenciando una heterogeneidad de actores en situación asimétrica de capacidades y recursos; además de una multiplicidad de condiciones ambientales que, desde luego, deberían ser tomadas en cuenta en la implementación de los programas. Dicha territorialización debería basarse en aspectos ambientales, socioeconómicos y culturales, con adecuaciones a las necesidades específicas de los grupos sociales y tomando en cuenta su cosmovisión. Dado que las comunidades locales y los pueblos indígenas de México son actores fundamentales para la conservación de la enorme riqueza cultural y natural de nuestro país (Suarez, 2017).

El acceso a la información y a la participación son dos puntos fundamentales para el fortalecimiento de los programas. Si bien el acceso a la información es un derecho regulado por ley, aún es bastante débil en términos de la promoción y ejercicio de derechos. De hecho, las poblaciones de territorios rurales, en donde se ubican los bosques, ni siquiera conocen su derecho de acceso a la información, por lo cual los programas deben fortalecer la difusión del acceso a la información en un marco de promoción del ejercicio de derechos, rompiendo la práctica vertical de unilateralidad de los responsables del programa a la ciudadanía, hacia la creación de una verdadera plataforma de diálogo horizontal. La participación permite a la ciudadanía conocer e involucrarse en el proceso de toma de decisiones que determinan las acciones a llevar a cabo por las instituciones del Estado. Brinda los instrumentos que permiten a los actores sociales tener mayor control sobre la gestión pública. En estos términos la participación, además de constituir un objetivo en sí, también es un medio para fortalecer la relación de la ciudadanía con el poder político y reforzar la confianza de la población en la Administración pública (Tornos Mas et al., 2012).

Finalmente, hace falta un fortalecimiento del proceso de monitoreo de los programas. El monitoreo es un mecanismo de información, seguimiento y control sobre sus actividades y resultados, por lo tanto, permite una supervisión continua o periódica de su ejecución para verificar tanto el avance en términos ambientales (proceso físico de reforestación, construcción de presas para control de cárcavas, etc.), como su impacto social (proceso sociocultural), los cuales abonan a fortalecer los mecanismos de transparencia y rendición de cuentas de los programas e impulsar su apropiación a nivel local. Por ello, constituye un sistema de información y análisis, orientado a identificar medidas correctoras frente a desviaciones o deficiencias, proponer alternativas cuando sea necesario (actividades no previstas) y recoger enseñanzas útiles (lecciones aprendidas) para el diseño de futuras intervenciones. Mediante el monitoreo se determina el grado en que se alcanzan los objetivos propuestos, la necesidad de modificar algunas acciones, los cambios que se deben buscar y sus porqués. Según Cotler et al. (2007), los gobiernos no pueden eludir su obligación de formular políticas de conservación amparadas en procesos de investigación, difusión, capacitación, seguimiento y comunicación constante con los actores involucrados, de quienes depende el éxito o fracaso de su aplicación en los territorios.

\section{Conclusiones}

La investigación estuvo enfocada a reflexionar sobre la relación de actores locales de la microcuenca Ichupio con sus bosques desde tres ámbitos: acceso y uso del bosque; impacto de las acciones de conservación de bosques, llevado a cabo en la zona; y estrategias a implementarse en los programas de recuperación ambiental para que puedan contribuir a responder a las demandas locales y así tener mayor impacto positivo en la conservación de los bosques. La reflexión se da a partir del estudio de las percepciones ambientales de la población de la zona de estudio, debido a que el acercamiento a las percepciones brinda información acerca de la lógica subjetiva de la interacción entre actores locales y sus bosques, lo que permite una lectura de los intereses y necesidades de conservación desde la propia voz de las personas, su cultura y valores. Desde luego, ello constituye un insumo esencial para mejorar las intervenciones de las distintas instancias orientadas a la restauración y conservación de bosques, al brindar elementos que permitan compatibilizar los intereses y objetivos de los programas con las preferencias y demandas sociales.

En lo referente al acceso y uso del bosque, se vislumbran dos problemáticas en la microcuenca de Ichupio, las cuales concurren para que los bosques no puedan desarrollar un rol relevante tanto en términos ecológicos como económicos para la región, a saber: el conflicto potencial por la tenencia de la tierra con el municipio de Tzintzuntzan y la carencia de un plan de manejo forestal consensuado al interior de la localidad. Mientras la cabecera municipal siga haciendo uso del bosque de Ichupio, pero sin responsabilidades con su conservación, 
seguirá un conflicto potencial entre ambas localidades, lo que repercute directamente en la conservación de los recursos, dado que los comuneros de Ichupio se plantean la disyuntiva: ¿si ellos usan nuestro bosque y no hacen nada para conservarlo, por qué nosotros lo debemos hacer? Y, por otro lado, un plan de manejo forestal es un instrumento que asegura tanto la conservación de la biodiversidad del bosque, manteniendo los procesos y funciones del bosque, como la producción de bienes y servicios a partir de los ecosistemas, preservando e incrementando sus potencialidades, en un marco de satisfacción de las necesidades sociales; y su ausencia dificulta que se logre una armonía entre las interacciones ecológicas, económicas y sociales en la región, a mediano y largo plazo.

Con relación al impacto de las actividades de conservación de suelo y agua en la microcuenca Ichupio, llevadas a cabo en el marco del programa gubernamental de recuperación ambiental de la cuenca del lago de Pátzcuaro, se reconoce que las acciones de reforestación y de construcción de presas para el control de cárcavas ha favorecido la revegetación y limitado el crecimiento de las cárcavas intervenidas, limitando el azolve del lago de Pátzcuaro; sin embargo, la degradación del bosque persiste. Por otra parte, la población local percibe debilidades importantes en el desarrollo del proceso, entre ellas: exclusión de la población y sus intereses y conocimientos en la toma de decisiones de las acciones; falta de coherencia entre los tiempos ambientales y burocráticos, con la entrega atrasada de plantas, en periodos en donde ya se compromete el resultado de la reforestación; siembra en lugares poco favorables, complicando el resultado esperado y falta de seguimiento de las actividades.

La participación local ha sido principalmente por incentivos y se ha centrado en la etapa de ejecución, lo que limita el involucramiento efectivo con el seguimiento de las acciones. Si bien la población reconoce el incentivo económico como un recurso que ayuda a la economía familiar, los actores locales no han jugado un papel determinante en la toma de decisiones y las acciones de conservación no ha tenido un impacto concreto en las condiciones de vida de la población y en su relación con los bosques.

Los resultados encontrados invitan a una reflexión sobre la compleja relación entre las intervenciones realizadas con el objetivo de recuperar los bosques y las poblaciones locales. Las percepciones sociales sobre la problemática de los bosques pueden incidir para reorientar las estrategias de conservación desde, al menos, dos perspectivas: a) permite la generación de alternativas concretas que dan solución a los problemas específicos de los territorios, y b) puede ayudar a reducir la brecha entre los postulados teóricos de los programas y su instrumentación en la práctica. Replantear las estrategias de los programas desde la perspectiva de atacar las causas de la problemática y no solamente sus síntomas; territorializar los diagnósticos y promover la participación en la toma de decisiones; así como generar un proceso robusto de seguimiento, en aras de asegurar los resultados esperados es un gran reto, sin embargo, es imprescindible que se avance en este sentido, pues solo de esta manera se logrará cumplir con el mandato de la conservación de los recursos.

\section{Contribuciones de los autores}

- Susana Ortega López: conceptualización, investigación, metodología, administración del proyecto, redacción - borrador original, redacción - revisión y edición.

- Denise Soares de Moraes: investigación, metodología, administración del proyecto, redacción -borrador original, redacción - revisión y edición.

\section{Referencias}

Aguirre-Calderón, O. A. (2015). Manejo Forestal en el Siglo XXI. Madera y Bosques, 21(especial), 17-28. https://doi.org/10.21829/myb.2015.210423

Amézcua Luna, J., y Sánchez Díaz, G. (2015). P’urhépecha. Pueblos Indígenas de México en el Siglo XXI. Comisión Nacional para el Desarrollo de los Pueblos Indígenas [CDI]. https://www.gob.mx/cms/uploads/ attachment/file/189198/cdi-monografia-purhepecha.pdf

Argueta, A., y Castilleja, A. (2008). El agua entre los p'urhépecha de Michoacán. Cultura y representaciones sociales, 3(5), 64-87. http://www.culturayrs.unam.mx/index.php/CRS/article/view/536/543

Arizpe, L., Paz Salinas, M. F., y Velázquez, M. (1993). Cultura y cambio global: percepciones sociales sobre la deforestación en la Selva Lacandona. Universidad Nacional Autónoma de México, Centro Regional de Investigaciones Multidisciplinarias Miguel Ángel Porrúa. 
Ávila García, P., Manrique Ascencio, D., García Calderas, S., Luna Sánchez, E., y Alcocer Almaraz, A. G. (2014). Producto 1. Evaluación Social Regional. Sistema de Microcuenca prioritarias "Pátzcuaro - Zirahuén”. SEMARNAT, CONAFOR y CIEco-UNAM. http://sis.cnf.gob.mx/wp-content/plugins/conafor-files/2018/nacional/catalogo/biblioteca/80.pdf

Bertoni, M., y López, M. J. (2010). Percepciones sociales ambientales: valores y actitudes hacia la reserva de Biósfera "Parque Atlántico Mar Chiquita" Argentina. Estudios y Perspectivas en Turismo, 19(5), 835849. https://www.estudiosenturismo.com.ar/PDF/V19/v19n5a14.pdf

Chanca Flores, A., Ávila Mendoza, J., y Aliaga Caynicela, M. Y. (2020). Percepción cultural de los pobladores sobre el Programa Social Juntos en el Valle del Mantaro. Socialium, 4(1), 68-83. https://doi. org/10.26490/uncp.s1.2020.4.1.510

Chávez-León, G., Tapia Vargas, L. M., Bravo Espinoza, M., Sáenz Reyes, J. T., Muñoz Flores, H. J., Vidales Fernández, I., Larios Guzmán, A., Rentería Ánima, J. B., Villaseñor Ramírez, F. J., Sánchez Pérez, J. L., Alcántar Rocillo, J. J., y Mendoza Cantú, M. (2012). Impacto del cambio de uso de suelo forestal a huertos de aguacate. Instituto Nacional de Investigaciones Forestales, Agrícolas y Pecuarias [INIFAP], Centro de Investigación Nacional Pacifico Centro, Campo Experimental Uruapan.

Comisión Forestal del Estado de Michoacán [COFOM]. (2007). Programa de Desarrollo Forestal Sustentable del Estado de Michoacán 2030. Tomo I. COFOM http://www.conafor.gob.mx:8080/documentos/docs/12/182Programa\%20Estrat\%c3\%a9gico\%20Forestal\%20de1\%20Estado $\% 20 \mathrm{de} \% 20$ Michoacan\%20Tomo\%20I.pdf

Comisión Nacional Forestal [CONAFOR]. (2019). El sector Forestal Mexicano en Cifras 2019. Bosques para el bienestar social y climático. Coordinación de Apoyo y Proyectos Especiales y Gerencia de Comunicación y Producción de CONAFOR. https://www.gob.mx/conafor/documentos/el-sector-forestal-mexicano-en-cifras-2019

Cotler, H., Sotelo, E., Domínguez, J., Zorrilla, M, Cortina, S., y Quiñones, L. (2007). La conservación de suelos: un asunto de interés público. Gaceta Ecológica, 83, 5-71. https://www.redalyc.org/pdf/539/53908302.pdf

Cruz Angón, A., Melgarejo, E. D., Camacho Rico, F., y Nájera Cordero, K. C. (coords.). (2013). La biodiversidad en Chiapas: Estudio de Estado. Comisión Nacional para el Conocimiento y Uso de la Biodiversidad [CONABIO]. https://doi.org/10.5962/bhl.title.118638

Del Castillo, A. (2020). México: el oasis forestal purépecha que surgió de las cenizas del volcán. Mongabay. Periodismo Ambiental Independiente Latinoamérica. https://es.mongabay.com/2020/07/mexico-el-oasis-forestal-purepecha/

Díaz-Bravo, L., Torruco-García, U., Martínez-Hernández, M., y Varela-Ruiz, M. (2013). La entrevista, recurso flexible y dinámico. Investigación en Educación Médica, 2(7), 162-167. http://riem.facmed. unam.mx/node/47

Dodds, W. K., Perkin, J. S., y Gerken, J. E. (2013). Human impact on freshwater ecosystem services: A global perspective. Environmental Science and Technology, 47(16), 9061-9068. https://doi. org/10.1021/es4021052

Durand, L. (2008). De las percepciones a las perspectivas ambientales. Una reflexión teórica sobre la antropología y la temática ambiental. Nueva Antropología, 21(68),75-87. https://revistas-colaboracion.juridicas. unam.mx/index.php/nueva-antropologia/article/view/15914

Endara Agramont, A. R., y Herrera Tapia, F. (2016). Deterioro y conservación de los bosques del Nevado de Toluca y el rol de los actores locales. Ciencia ergo-sum, 23(3), 247-254. https://cienciaergosum. uaemex.mx/article/view/7367

Feria Zapata, R., Villamil González, A. L., y Vergara Escobar, O. (2019). Evaluación de la percepción de la educación en promoción de salud y estilos de vida de estudiantes de la Universidad de Tolima. Revista Chilena de Enfermeria, 1(1), 20-32. https://doi.org/10.5354/2452-5839.2019.55438

Fernández Moreno, Y. (2015). ¿Por qué estudiar las percepciones ambientales?: Una revisión de la literatura mexicana con énfasis en Áreas Naturales Protegidas. Espiral Estudios Sobre Estado y Sociedad, 15(43), 179-202. http://espiral.cucsh.udg.mx/index.php/EEES/article/view/1378

García Villanueva, N. H. (ed.). (2009). Memoria ilustrada del Programa para la Recuperación Ambiental de la Cuenca del Lago de Pátzcuaro: Avances (2003-2008). IMTA.

Gómez Durán, T. (2020). México: desdén presupuestal, deforestación y defensores asesinados. Mongabay. Periodismo Ambiental Independiente Latinoamérica. https://es.mongabay.com/2020/01/mexico-balance-deudas-ambientales-2019/ 
Hensler, L., y Merçon, J. (2020). Áreas Naturales Protegidas como territorios en disputa: intereses, resistencias y acciones colectivas en la gestión compartida. Sociedad y Ambiente, 22, 180-211. https://doi. org/10.31840/sya.vi22.2101

Instituto Mexicano de Tecnología del Agua [IMTA], y Fundación Gonzalo Río Arronte. (2004). Descubre una cuenca: el Lago de Pátzcuaro. IMTA y Fundación Gonzalo Río Arronte.

Instituto Mexicano de Tecnología del Agua [IMTA]. (2011). La reforestación y la participación social en la cuenca del lago de Pátzcuaro, Michoacán. IMTA. Informe Inédito.

Instituto Nacional de Estadística y Geografía [INEGI]. (2014). Guía para la interpretación de Carta Edafológica. Escala 1: 250 000. Serie III. INEGI https://www.inegi.org.mx/contenidos/productos/prod_serv/ contenidos/espanol/bvinegi/productos/nueva_estruc/702825076221.pdf

Instituto Nacional de Estadística y Geografía [INEGI]. (2020). Censo de Población y Vivienda 2020. Principales resultados por localidad (ITER). INEGI. https://www.inegi.org.mx/programas/ccpv/2020/

Jiménez Pérez, J. (2009). Reforestación. Evaluación Externa. Ejercicio Fiscal 2008. SEMARNAT, CONAFOR, Universidad Autónoma de Nuevo León.

Lazos Chavero, E., y Paré Ouellet, L. (2000). Miradas indígenas sobre una naturaleza entristecida: Percepciones del deterioro ambiental entre nahuas del sur de Veracruz. Plaza y Valdés. http://ru.iis.sociales. unam.mx/handle/IIS/5075

Millán Malo, G. C. (2005). La comunicación en la gestión integral de cuencas. Encuentro por una Nueva Cultura del Agua en América Latina. https://agua.org.mx/wp-content/uploads/2010/10/639la_comunicacion_en_la_gestion_integral_de_cuencas.pdf

Monforte, G. G., y Cantú, M. P. C. (2009). Escenario del agua en México. Cultura Cientifica y Tecnológica, 6(30), 31-40. http://erevistas.uacj.mx/ojs/index.php/culcyt/article/view/356

Peralta-Rivero, C., Galindo-Mendoza, M. G., Contreras-Servín, C., Algara-Siller, M., y Mas-Caussel, J. F., (2016). Percepción local respecto a la valoración ambiental y pérdida de los recursos forestales en la región Huasteca de San Luis Potosí, México. Madera y bosques, 22(1), 71-93. https://doi. org/10.21829/myb.2016.221478

Pérez Andrés, C. (2002). Sobre la metodología cualitativa. Revista Española de Salud Pública, 76(5), 373-380. https://recyt.fecyt.es/index.php/RESP/article/view/769

Programa Mundial de las Naciones Unidas de Evaluación de los Recursos Hídricos [WWAP]. (2018). Informe mundial de las Naciones Unidas sobre el desarrollo de los recursos hidricos 2018: soluciones basadas en la naturaleza para la gestión del agua. UNESCO. https://unesdoc.unesco.org/ark:/48223/pf0000261494

Santika, T., Wilson, K. A., Budiharta, S., Kusworo, A., Meijaard, M., Law, E. A., Friedman, R., Hutabarat, J. A., Indrawan, T. P., St. John, F. A. V., y Struebig, M. J. (2019). Heterogeneous impacts of community forestry on forest conservation and poverty alleviation: Evidence from Indonesia. People and Nature, 1(2), 204-219. https://doi.org/10.1002/pan3.25

Secretaría de Medio Ambiente y Recursos Naturales [SEMARNAT]. (2016). Informe de la Situación Medio Ambiente en México. Compendio de Estadísticas Ambientales. Indicadores clave, de desempeño ambiental y de crecimiento verde. Edición 2015. SEMARNAT. https://apps1.semarnat.gob.mx:8443/dgeia/ informe15/tema/pdf/Informe15_completo.pdf

Sentencia pronunciada en el expediente 423/02, relativo al reconocimiento y titulación de bienes comunales del núcleo indígena de Tzintzuntzan, Municipio de Tzintzuntzan, Michoacán. Tribunal Unitario Agrario. Diario Oficial de la Federación del 06 de noviembre de 2014, Segunda Edición. https://www.dof.gob.mx/ nota_detalle.php? codigo $=5367288 \&$ fecha $=06 / 11 / 2014$

Suarez, G. (2017). Las comunidades indígenas, custodias de los bosques y la biodiversidad. Consejo Civil Mexicano para la Silvicultura Sostenible A.C. Comunidades fuertes, territorios vivos. https://www.ccmss.org.mx/las-comunidades-indigenas-custodias-los-bosques-la-biodiversidad/

Suarez, G. (2019). Cheran, la lucha para recuperar el bosque. Consejo Civil Mexicano para la Silvicultura Sostenible A.C. Comunidades fuertes, territorios vivos. https://www.ccmss.org.mx/cheran-la-lucha-para-recuperar-el-bosque/

Tarannum, F., Kansal, A. y Sharma, P. (2018). Understanding public perception, knowledge and behaviour for water quality management of the river Yamuna in India. Water Policy, 20(2), 266-281. https://doi. org/10.2166/wp.2018.134

Tornos Mas, J., Arroyo Díez, A., Martínez Martínez, M., y López Pagán, J. (2012). Transparencia, rendición de cuentas y participación: una agenda común para la cohesión social y la gobernanza en América Lati- 
na. Diputación de Barcelona (Oficina de Coordinación y Orientación del Programa URB-AL III). https:// www.observ-ocd.org/sites/observ-ocd.org/files/2018-04/transparencia-rendicion-de-cuentas-y-participacion-una-agenda-comun-para-la-cohesion-social-y-la-gobernanza-en-america-latina.pdf

Torres-Rojo, J. M., Moreno-Sánchez, R., y Mendoza-Briseño, M.A. (2016). Sustainable Forest Management in Mexico. Current Forestry Reports, 2, 93-105. https://doi.org/10.1007/s40725-016-0033-0

Tuan, Y. -F. (1974). Topophilia: A Study of Environmental Perception. Attitudes and Values. Prentice-Hall.

Vargas Melgarejo, L. M. (1994). Sobre el concepto de percepción. Alteridades, 4(8), 47-53. https://alteridades. izt.uam.mx/index.php/Alte/article/view/588

Vargas Velásquez, S. (2014). Gobernanza ambiental del lago de Pátzcuaro y la pesca. En R. I. Huerta Delgadillo y S. Vargas Velásquez, Estudio Ecosistémico del lago de Pátzcuaro: Aportes en gestión ambiental para el fomento del desarrollo sustentable (Vol. II) (pp. 175-193). Instituto Mexicano de Tecnología del Agua, Comisión Nacional del Agua, Secretaría de Medio Ambiente y Recursos Naturales. https://www. imta.gob.mx/biblioteca/libros_html/lago-patzcuaro/files/assets/basic-html/page177.html

Zerpa de Kirby, Y. B. (2016). Lo cualitativo, sus métodos en las ciencias sociales. Sapienza Organizacional, 3(6), 207-230. https://www.redalyc.org/articulo.oa?id=553056828013

Zúñiga, I., y Deschamps, P. (2013). Políticas y subsidios forestales en México. Consejo Civil Mexicano para la Silvicultura Sostenible A.C. https://www.ccmss.org.mx/wp-content/uploads/CCMSS_Subsidios_Forestales_190513.pdf 